\title{
EDITORIAL
}

\section{Facing the change together: reflections of coping and resilience from American geriatric psychiatrists during COVID-19*}

\author{
"Now this is not the end. It is not even the beginning of \\ the end. \\ But it is, perhaps, the end of the beginning."
}

Winston Churchill (1942)

Over the past 2 months, we have witnessed Coronavirus Disease 2019 (COVID-19), the disease caused by the novel pathogen SARS-CoV-2, devastate the health of citizens across the world and paralyze the economy of the many countries, including the United States (US). Disasters disproportionately affect poor and vulnerable populations, and older adults with mental illness are at higher risk for the physical complications and psychiatric sequelae. The psychological impacts of COVID-19 and associated quarantine can range from short-term boredom and loneliness to longterm anxiety, depression, and post-traumatic stress disorder symptoms in vulnerable individuals (Brooks et al., 2020; Xiang et al., 2020). Lockdowns may also exacerbate pre-existing mental illness and limit access to timely medical care.

The US has the most confirmed cases of COVID-19 in the world at the time of writing this article (Johns Hopkins University, 2020). Geriatric nurses, nursing assistants, psychologists, social workers, physical/occupational/speech/recreational therapists, environmental and food services technicians, nurse practitioners, physician assistants, and psychiatrists are at the forefront of safeguarding the mental health of senior American adults for whom COVID-19 may be most psychologically taxing. In response, the pandemic has quickly altered nearly all aspects of routine healthcare delivery including inpatient, outpatient and long-term care. Extensive changes are also being encountered in specific treatment interventions such as the administration of ECT and prescribing practices for certain vital medications like clozapine (Siskind et al., 2020; Clozapine REMS, 2020).

\footnotetext{
* This article has been simultaneously published with permission in International Psychogeriatrics and The American fournal of Geriatric Psychiatry.
}

Geriatric psychiatrists across the US engaged in an extensive, informal series of communications during the earliest days of the COVID-19 pandemic. Conversations largely focused on how to best adapt clinical services to digital platforms while maintaining quality of graduate medical education. Here we present a thematic analysis of these digital correspondences. The following quotes and insights were not selected based on any formal methodological approach and do not reflect a comprehensive representation of formal geriatric psychiatry leadership. Rather, this article captures an ad hoc series of voices and opinions of how geriatric psychiatrists may face the numerous challenges of COVID-19 together.

\section{Rapid implementation of telepsychiatry}

Geriatric psychiatrists play a critical role in ensuring the continued mental health treatment of elderly patients despite physical distancing. The pandemic has catalyzed the adoption of telepsychiatry at record speed as physical distancing becomes the new norm. Key administrative issues for implementation include licensure requirements; malpractice insurance; status of insurance coverage for virtually delivered services; adherence to confidentiality and security regulations; and establishment of protocols for managing laboratory tests, prescriptions, and scheduling (Wright and Caudill, 2020). However, regulatory bodies have assisted by temporarily waiving numerous privacy policies around telepsychiatry on March 17, 2020 (Centers for Medicare \& Medicaid Services, 2020).

Adapting mental healthcare delivery systems is especially challenging in a decentralized healthcare system like the US where each state has unique legislative and Medicaid reimbursement approaches to COVID-19. Medicare is the largest insurer for older Americans, but Medicare did not reimburse psychiatry for telemedicine prior to COVID-19. This was literally a non-starter for telemedicine in a fee-for-service setting for older patients. The 
necessity to evolve reimbursement models during COVID has led Medicare to allow reimbursement for telemedicine for the first time. This has been a particularly monumental step towards widespread implementation and sustainability of telepsychiatry during and after the acute phases of the pandemic.

Heterogeneous healthcare systems are feeling a previously unimaginable impetus to expand their telehealth capacity. Stanford University was able to amazingly overcome years of regulatory and technological hurdles to transition to telepsychiatry within a week. Rates of expansion, however, largely depend on pre-COVID functionality. Systems conducting virtual visits beforehand were able to expand upon existing protocols for the pandemic. Other practices were already using an electronic medical record (EMR) with a pre-existing, readily activated, embedded platform for virtual visits. Otherwise, independent digital conferencing platforms like Zoom or VIDYO are being used to conduct virtual visits while documenting in a separate EMR. Systems still utilizing paper charts, however, are experiencing a slower transitioning process. In these instances, providers cannot access charts to review in a remote treatment situation. Therefore, it has been necessary to create protocols for retrieving and returning relevant information to the remote location.

\section{Disadvantaged populations without access to technology}

Many older adults face a steep technological learning curve or simply do not have the resources for videoconferencing. Practice sites are substituting calling the patient and/or family member by phone when video technology is not feasible. Other creative workarounds facilitate in-person, "virtual" visits for disadvantaged clients. Patients may come in-person to a designated room near the entrance of the outpatient clinic that houses a computer monitor already set up to virtually speak to the remote provider. This alleviates patients of the need to troubleshoot technology while maintaining decreased physical exposure. Staff clean the room after each visit. An alternate successful strategy on inpatient units or long-term care facilities is to have staff take a sanitized laptop or mobile device directly to the patients. Devices are cleaned between each patient.

\section{Workflow of Virtual Visits}

One first step of conducting virtual visits is to make sure that patients and staff have access to clearly written directions for use of digital technologies. It has been found helpful to develop a series of workflow instructions for both providers and patients with screenshots and instructions for desktop and mobile device access. It may also be beneficial to reach out to patients ahead of the appointment to troubleshoot connectivity issues and how to work with clinic staff.

The pace of virtual consults generally mirrors that of in-person appointments. McLean Hospital staff and psychiatrists call family members after the initial patient visit to collect additional historical information, review current assessment, and plan. Some hospital-based consultations are not utilizing videoconferencing at all. At the Tripler Army Medical Center in Hawaii, medical colleagues including emergency physicians contact psychiatrists, including geriatric psychiatrists, who review the EMRs and provide phone consultations. If necessary, patients may be interviewed using a phone with the assistance of an emergency room staff member. However, sometimes in person interview is done while using appropriate personal protective equipment.

\section{Special aspects and limitations of virtual visits}

Managing the therapeutic milieu is proving challenging with virtual visits. From a technological perspective, the viewing area of the camera lens may restrict how much of the patient and their surroundings may be seen. It was also noted that the device microphone and speakers may be insufficient for some patients with soft voices or dysarthric speech. Ambient and background noises may exacerbate difficulties hearing patients. Establishing rapport with patients experiencing psychosis or paranoia may be problematic when relying upon technology. Furthermore, some clinicians felt an increased level of intimacy when videoconferencing with patients in their personal homes versus a more neutral clinic setting. Some providers even expressed that having patients view the inside of their homes during visits felt somewhat intrusive.

The practice of electroconvulsive therapy (ECT) has been modified and limited by the need for personal protective equipment for droplet precautions and the need to maintain physical distancing through telehealth (Colbert et al., 2020). McLean Hospital has reduced its volume of ECT by about $30 \%$ by restricting its use to hospitalized inpatients with acute needs and outpatients undergoing maintenance treatments. If the clinical situation of the patients permits, they are attempting to substitute the missing ECT treatments with less invasive modalities like transcranial magnetic stimulation 
and ketamine. In other instances, physical distancing and reliance upon virtual visits has required cancellation of all but essential operations. At Stanford University and Saint Louis University, ECT has been primarily reduced to patients receiving court-ordered ECT, inpatients, and emergencies.

\section{COVID-19 and social isolation for patients with dementia and delirium}

Physical and social isolation from caregivers and family members poses challenges for individuals with delirium and dementia during the COVID-19 pandemic (Wang et al., 2020). The presence of family and friends is well-known to benefit patients coping with disorientation, thus healthcare providers are busier than ever while compensating for patients' lack of family support. The pandemic is also creating increased patient acuity and/or volumes that frontline providers must treat. Moreover, the presence of masks impedes facial recognition and interpersonal reassurance to those patients who desperately need to reorient.

The psychiatric needs of our patients with dementia and delirium are greater than ever. The primary indications of new inpatient and/or nursing consults largely reflect these elevated needs. Indeed, the primary indications for psychiatry consults at the Tripler Army Medical Center in Hawaii include depression, anxiety, dementia, delirium, other neuropsychiatric disorders, decisional capacity evaluations, and end of life issues. Virtual consults have certain drawbacks, however. Executive function tests administered by pen and paper may be less feasible when virtually performing cognitive examinations for patients with dementia or delirium. Online versions of these tests are available to bypass this limitation should patients have access to a compatible device.

Healthcare workers are wholeheartedly seeking to go the distance to address these new needs of our patients. Many long-term care facilities are making special efforts to digitally connect patients with their loved ones by facilitating video chats and by posting resident pictures daily on a private Facebook site. Special efforts are also being made to check on the wellbeing of patients' families. In recognition of the efforts of our colleagues, mental health workers are banding together to provide additional support to frontline staff. For example, some faculty at Stanford University are reported to be volunteering their services as peer supports to exhausted frontline providers.

\section{Redeployment of staff and repurposing of clinical facilities}

Redeployment of some psychiatrists and residents across the US has necessitated a broader scope of practice, especially in the most affected areas. Mount Sinai Hospital is located in New York City, America's most impacted city. Trainees at Mt. Sinai may be sent to various clinical areas depending on staffing needs. This is typically first to psychiatry primary services but may also include sending trainees to medical units. Fellows may be called to function as attendings in their primary specialities. Stanford University is in a relatively less affected area than New York City. There, residents and fellows were not redeployed until several weeks after the beginning of the pandemic. Others, like UConn Health in central Connecticut and Saint Louis University in Missouri, have not been redeployed at all as of mid-April 2020. Nonetheless, contingency plans are being designed throughout the country should the need arise to lend staffing support to medical specialties while continuing appropriate psychiatric care.

In addition to redeployment of staff, there has been conversion of hitherto psychiatric or rehabilitation facilities. In some instances, both general psychiatry and geriatric psychiatry units are being converted to single patient rooms only to allow for maximum physical distance between patients. An unfortunate consequence is that overall capacity on inpatient units decreases. Other units are being repurposed to expand space for COVID-19 patients. For example, rehabilitation facilities may be converted to treat COVID-19 patients with mild illness or those in convalescence. Concerns arise about how to best protect vulnerable and elderly nursing home residents. Staff protection during unit repurposing is also a noted concern, such as when personal faculty offices are being used to hold meetings without masks. More broadly, we have seen recommendations around geriatric and long-term care facilities drafted and re-drafted as our colleagues wrangle with the fluidity and rapid evolution of the pandemic (Ouslander, 2020).

\section{Residency education}

Resident education continues in some form in most states of the US, but it appears to vary according to the degree of severity of COVID-19 in that geographic region. Across the country, face to face meetings have been cancelled. At Mt. Sinai, graduate medical education is in an "emergency 
pandemic status" in areas that are hardest hit, like New York City. All classes and lectures have been suspended there. Didactic curriculum teaching is proceeding in modified fashion by Zoom in less impacted areas. Zoom is also replacing in person interactions for morning resident teaching rounds, supervision, lectures and meetings. The Teaching and Training Committee of the American Association for Geriatric Psychiatry is developing a series of didactic lectures that will be posted on the American Association for Geriatric Psychiatry website for residents during their geriatric psychiatry clinical rotation.

\section{Patient and provider satisfaction during COVID-19}

Overall, patients and their families have been receptive to the use of these unfamiliar technologies as a means of unifying efforts to combat the pandemic. Many providers also reported feeling satisfied with their ability to provide time-efficient, quality mental healthcare from a digital platform. Providers express being able to complete most aspects of the mental status examination and a modified neurological examination with observation of gait, posture, facial expression and upper extremity tremor/lower extremity movements. Some aspects of psychiatric care, however, cannot be replicated without person to person contact. Subtle differences in body language, facial expressions, and slight vocals alterations are far more difficult to be detected using digital technologies.

The practice limitations during COVID-19 have led to some deep self-reflections amongst America's most senior psychiatrists. For some, a nostalgia for pre-COVID, in-person patient care leads to contemplations of early retirement should the field require a prolonged time to reinstate person to person care as previously. Other providers on inpatient services expressed a desire to join their colleagues in delivering in-person patient care while yet being unable to do so due to personal health risk factors and/or age. In all cases, the sense of kinship and solidarity remains.

\section{Conclusion}

We are in the very early days of adapting personally and professionally to COVID-19. We write with trepidation at what lies ahead but also with a sense of camaraderie and kinship as we know we face this challenge united. It will be imperative that geriatric psychiatrists continue to demonstrate altruism, resilience, solidarity, hope, perseverance, and flexibility during these difficult times. We are buoyed that geriatric psychiatrists continue to electronically traverse geographical borders and are collaborating to prepare, plan, and deliver the best possible care to their patients and frontline colleagues. Furthermore, we continue to educate the next generation of health professionals to the best of our abilities during this time.

Ongoing international and interdisciplinary collaboration will be essential. It will also be essential that we advocate for older Americans who are at elevated risk of being marginalized and experiencing direct and indirect harm from COVID-19. As we continue to work together through this pandemic, we must remind colleagues and broader society of the adage popularized by the $16^{\text {th }}$ US Surgeon General, Dr. David Satcher: "there is no health without mental health."

\section{Acknowledgments}

The authors wish to recognize all of the contributors to this amazing correspondence chain. Thank you to Drs. Jahnavi Handa, Paul Kirwin, Iqbal Ahmed, Craig Nelson, Elliot Stein, Shilpa Srinivasan, Laura Dunn, Melinda Lantz, Sanford Finkel, David Steffens, George Grossberg, Joel Streim, Henry Brodaty, and Stephan Arndt. This perspective would not have been possible without your tireless dedication and input.

\section{Disclosures}

The authors have no conflicts of interest to declare.

Carmen Black Parker, ${ }^{1}$ (1) Malcolm P Forbes, ${ }^{2}$ IPSit V VAhia, ${ }^{3}$ BRent P Forester, ${ }^{3}$ Dilip V Jeste ${ }^{4}$ and Charles F Reynolds ${ }^{5}$

${ }^{1}$ Yale University School of Medicine, Connecticut Mental Health Center, New Haven, CT, USA

${ }^{2}$ Department of Psychiatry, The University of Melbourne, Melbourne, Australia

${ }^{3}$ Division of Geriatric Psychiatry, McLean Hospital, Harvard Medical School, Boston, MA, USA

${ }^{4}$ University of California, San Diego, CA, USA

${ }^{5}$ University of Pittsburgh, Pittsburgh, PA, USA

Email: carmen.parker@yale.edu

\section{References}

Brooks, S. K. et al. (2020). The psychological impact of quarantine and how to reduce it: rapid review of the evidence. The Lancet, 395(10227), 912-920.

Centers for Medicare \& Medicaid Services (2020, March 17). Medicare Telemedicine Health Care Provider 
Fact Sheet. Available at: https://www.cms.gov/ newsroom/fact-sheets/medicare-telemedicine-health-careprovider-fact-sheet; last accessed 10 April 2020.

Churchill, W. (1942). "The End of the Beginning." Speech at The Lord Major's Luncheon, Mansion House. Available at: http://www.churchill-society-london.org.uk/EndoBegn .html; last accessed 10 April 2020.

Clozapine REMS (2020). Important Program Update 04/02/ 2020. Available at: https://www.clozapinerems.com/ CpmgClozapineUI/home.u\#; last accessed 10 April 2020.

Colbert, S. A., McCarron, S., Ryan, G., and

McLoughlin, D. M. (2020). Images in clinical ECT: immediate impact of COVID-19 on ECT practice. The fournal of ECT, doi: 10.1097/YCT .0000000000000688 .

Johns Hopkins University (2020). Coronavrius COVID-19 Global Cases by the Center for Systems Science and
Engineering: Johns Hopkins University. Available at: https://coronavirus.jhu.edu/map.html; last accessed 10 April 2020.

Ouslander, J. G. (2020). Coronavirus-19 in geriatrics and long-term care: an update. Fournal of the American Geriatrics Society, doi: 10.1111 /jgs. 16464 .

Siskind, D. et al. (2020). Consensus statement on the use of clozapine during the COVID-19 pandemic. Fournal of Psychiatry E Neuroscience, 45(4), 200061.

Wang, H. et al. (2020). Dementia care during COVID-19. Lancet, 395(10231), 1190-1191.

Wright, J. H. and Caudill, R. (2020). Remote treatment delivery in response to the COVID-19 pandemic. Psychotherapy and Psychosomatics, 89, 130-132.

Xiang, Y.-T. et al. (2020). Timely mental health care for the 2019 novel coronavirus outbreak is urgently needed. The Lancet Psychiatry, 7(3), 228-229. 